\title{
Guidelines for the management of patients on oral anticoagulants requiring dental surgery
}

\author{
D. J. Perry, T. J. C. Noakes ${ }^{2}$ and P. S. Helliwell ${ }^{3}$
}

The objective of these guidelines is to provide healthcare professionals, including primary care dental practitioners, with clear guidance on the management of patients on oral anticoagulants requiring dental surgery. The guidance may not be appropriate in all cases and individual patient circumstances may dictate an alternative approach.

\section{INTRODUCTION}

The guideline group was selected to be representative of UK based medical experts and patients. MEDLINE and EMBASE were searched systematically for publications in English from 1950-2006 using the key words: dental, surgery, oral and anticoagulants. The writing group produced the draft guidelines, which were subsequently revised by consensus by members of the Haemostasis and Thrombosis Task Force of the British Committee for Standards in Haematology. The guidelines were then reviewed by a sounding board of approximately 100 UK haematologists, the BCSH (British Committee for

${ }^{1-3}$ British Committee for Standards in Haematology and I"Department of Haematology, Box 234, Addenbrooke's Hospital, Hills Road, Cambridge, CB2 200; ${ }^{2}$ Derriford Hospital NHS Trust, Derriford Road, Plymouth, Devon, PL6 8DH: ${ }^{3}$ National Patient Safety Agency (NPSA), 4-8 Maple Street, London, W1T 5HD

${ }^{*}$ Correspondence to: Dr David J. Perry

Email: david.perry@addenbrookes.nhs.uk

\section{Refereed Paper}

Accepted 2 May 2007

DOI: $10.1038 /$ bdj.2007.892

${ }^{\circledR}$ British Dental Journal 2007; 203: 389-393
Standards in Haematology), the British Society for Haematology Committee, the British Dental Association (BDA), and the National Patient Safety Agency (NPSA) and comments incorporated where appropriate. Criteria used to quote levels and grades of evidence are as outlined in appendix 3 of the Procedure for Guidelines Commissioned by the BCSH (http://www.bcshguidelines.com/ process1.asp\#App3).

\section{SUMMARY OF KEY RECOMMENDATIONS}

1. The risk of significant bleeding in patients on oral anticoagulants and with a stable INR in the therapeutic range $2-4$ (ie $<4$ ) is very small and the risk of thrombosis may be increased in patients in whom oral anticoagulants are temporarily discontinued. Oral anticoagulants should not be discontinued in the majority of patients requiring outpatient dental surgery including dental extraction (grade A level Ib)

2. For patients stably anticoagulated on warfarin (INR 2-4) and who are prescribed a single dose of antibiotics as prophylaxis against endocarditis, there is no necessity to alter their anticoagulant regimen (grade C, level IV)

3. The risk of bleeding in patients on oral anticoagulants undergoing dental surgery may be minimised by:

a. The use of oxidised cellulose (Surgicel) or collagen sponges and sutures (grade B, level IIb)

b. 5\% tranexamic acid mouthwashes used four times a day for two days (grade A, level Ib). Tranexamic acid is not readily available in most primary care dental practices.

4. For patients who are stably anticoagulated on warfarin, a check INR is recommended 72 hours prior to dental surgery (grade A, level Ib)

5. Patients taking warfarin should not be prescribed non-selective NSAIDs and COX-2 inhibitors as analgesia following dental surgery (grade B, level III).

1. The risk of bleeding in anticoagulated patients undergoing oral surgery Dental surgery in anticoagulated patients is common and historically their management has been controversial following early reports of major 
bleeding in such individuals. ${ }^{1}$ Many of the early reports of haemorrhage associated with dental surgery during this period predated the standardisation of oral anticoagulant control by means of the INR. In 1954, the American Heart Association recommended a therapeutic range for oral anticoagulant therapy of a prothrombin time ratio (PTR) of 2-2.5 using human brain reagents. ${ }^{2}$ Later, the use of less sensitive commercial thromboplastins was not accompanied by a change in the target PTR ratio. Clinicians, therefore, administered larger doses of oral anticoagulants to achieve the target ratio, resulting in an increased incidence of haemorrhage. The development and introduction of the INR did not take place until $1983 .^{3}$

The risks of bleeding associated with dental extraction in individuals not receiving oral anticoagulants is approximately $1 \% .{ }^{4,5}$ In a review of ten studies of patients undergoing dental surgery and in whom oral anticoagulants were continued, 9\% (89/990) had delayed postoperative bleeding and in 3.5\% of cases this was classified as 'serious' ie not controlled by local measures. ${ }^{4}$ Other studies have reported the incidence of minor bleeding as higher and in some cases up to $50 \% .^{5}$ However, the interpretation and comparison of bleeding rates in patients undergoing oral surgery is difficult as rates for different procedures are not analysed separately, the definitions used to describe serious bleeding vary and surgery can involve the use of differing treatments to secure haemostasis.

\section{What constitutes dental treatment?}

Many procedures performed in the primary care setting are relatively noninvasive and would not, therefore, require measurement of the INR. Such procedures would include prosthodontics [construction of dentures], scaling/ polishing and some conservation work [fillings, crowns, bridges].

Potentially invasive procedures performed in primary care would include:

- Endodontics [root canal treatment]

- Local anaesthesia [infiltrations, inferior alveolar nerve block, mandibular blocks]

- Extractions [single and multiple]

- Minor oral surgery

- Periodontal surgery
- Biopsies

- Subgingival scaling.

3. Which patients on warfarin should NOT have a surgical dental procedure in the primary care setting?

- Patients on oral anticoagulants with co-existing medical problems eg liver disease, renal disease, thrombocytopenia or who are taking anti-platelet drugs. Such patients may have an increased risk of bleeding

- Patients requiring surgical procedures not listed above. Such patients should be referred to a dental hospital or hospital-based oral and maxillofacial surgery department.

Surgical dentists in the primary care setting often have the skills to undertake any of the procedures listed above in the context of the anticoagulated patient. The skill and experience of the primary care dentist, together with the difficulty of the procedure all need consideration.

\section{Is it safe to discontinue anticoagulants prior to dental surgery? \\ 4.1. The risk of thrombosis if anticoagulants are discontinued}

The risk of thrombosis associated with temporarily discontinuing anticoagulants prior to dental surgery is small but potentially fatal. In the review of Wahl, 5/493 (1\%) patients undergoing 542 dental procedures and in whom anticoagulants were withdrawn specifically for surgery, had serious embolic complications of which four were fatal. ${ }^{6}$ The four deaths comprised: a fatal cerebral embolism 17 days after discontinuing warfarin; one fatal myocardial infarction 19 days after interruption of therapy for nine days; one fatal cerebral thromboembolism five days postoperatively and one patient died from a thromboembolism but no other data are available.

\subsection{The risk of major bleeding in patients undergoing oral surgery if anticoagulants are continued}

Wahl reviewed 26 papers comprising 2,014 dental surgical procedures in 774 patients receiving continuous warfarin therapy, including 1,694 patients undergoing single, multiple extractions and full mouth extractions. ${ }^{6}$ This metanalysis included patients with an INR up to 4.0. Although some patients had minor oozing treated with local measures, more that 98\% of patients receiving continuous anticoagulants had no serious bleeding problems. Many of the procedures were performed in patients with an INR above the present recommended therapeutic levels of anticoagulation. Twelve patients $(<2 \%)$ had postoperative bleeding problems that were not controlled by local measures. Eight of these 12 episodes were associated with a supratherapeutic INR at the time of the procedure or in the week after. Major bleeding was rare (4/2012, $0.2 \%$ ) for patients with a therapeutic INR $(<4)$ undergoing dental surgery. There were no deaths.

Five randomised studies have been published since this systematic review. Devani et al. ${ }^{7}$ randomised 65 patients on continuous warfarin therapy and undergoing 133 dental extractions to either stop warfarin two to three days prior to surgery (32 patients) resulting in a reduction in mean INR from 2.6 to 1.6 , or to continue anticoagulants with no dose alteration (32 patients) and a mean pre-operative INR of 2.7 (range 2.2-3.9). All patients received local measures ('Surgicel' packing and sutures) to control post-operative bleeding. None of the patients had any immediate postoperative bleeding and only one patient from each group had mild delayed haemorrhage, which was controlled with local measures.

Campbell et al. randomised 25 patients to either discontinue warfarin 72-96 hours prior to surgery (13 patients: mean INR 2.0: range 1.2-2.9) or to continue anticoagulants (12 patients: mean INR 2.0: range 1.1-3.0). ${ }^{8} \mathrm{~A}$ further group of ten individuals not receiving anticoagulants served to establish a baseline bleeding risk. No patient in any group had postoperative bleeding 'sufficiently severe to require therapeutic intervention and there were no significant differences in blood loss between the three groups.

Evans randomised 109 patients on continuous oral anticoagulants requiring dental extractions to either continue warfarin (57 patients) although if the INR was $>4$ on the day of surgery, the patient was withdrawn. ${ }^{9}$ The control group (52 patients) stopped taking warfarin two days prior to surgery and if the INR was $>2$ on the day of the procedure this was subsequently rebooked for the following day. Tranexamic acid 
mouthwashes were not allowed. The mean INR for the group taking anticoagulants at the time of dental surgery was 2.5 (range 1.2-4.7) and for the group that discontinued anticoagulants 1.6 (range 1.2-2.3). The rate of bleeding was higher in the group continuing anticoagulation $(15 / 57,26 \%)$ than in the control group $(7 / 52,14 \%)$ but the difference was not significant.

Sacco et al..$^{10}$ randomised 131 patients on anticoagulants requiring dental surgery - primarily dental extractions - to either reduce their warfarin dose 72 hours prior to surgery to achieve an INR of 1.5-2 on the day of surgery, or to continue anticoagulants with no alteration, but this latter group received haemostatic agents at the time of surgery and tranexamic acid mouthwashes for the following six days. Bleeding sufficient to warrant additional haemostatic measures was observed in 15\% of the first group (mean INR $1.77 \pm 0.26$ ) and $9 \%$ of the second group (mean INR $2.89 \pm 0.42)$.

Al-Mubarak et al. randomised 168 patients on warfarin undergoing dental surgery to one of four groups: no socket suturing with or without discontinuation of warfarin and socket suturing with or without discontinuation of warfarin (groups 1-4 respectively). ${ }^{5}$ The percentage of patients who bled following surgery was 12\% in the group whose warfarin was stopped and who in addition had suturing of the socket (mean pre-operative INR of 1.79) compared to $36 \%$ in Group 4 with a mean pre-operative INR of 2.6 but whose warfarin was continued and who did not receive suturing of the sockets following extraction. However, this difference in bleeding was not clinically significant and no surgical intervention was necessary.

Recommendations: The risk of significant bleeding in patients on oral anticoagulants and with a stable INR in the therapeutic range 2-4 is low. The risk of thrombosis if anticoagulants are discontinued may be increased. Oral anticoagulants should not be discontinued in the majority of patients requiring outpatient dental treatment. An appreciation of the surgical skills of primary care dentists and the difficulty of surgery, particularly when INR levels approach 4 , is also important when assessing the risk of bleeding. Individuals in whom the INR is unstable should be discussed with their anticoagulant management team (grade A level Ib).

\section{Anticoagulants and prophylactic antibiotics}

Patients undergoing dental surgery may be prescribed antibiotics to prevent endocarditis. Antibiotics prescribed commonly include amoxicillin, ampicillin, clindamycin and azithromycin. ${ }^{11} \mathrm{~A}$ single dose of an antibiotic is unlikely to have any significant effect upon the INR. Individuals who are prescribed more than a single dose of antibiotics should have the INR measured two to three days after starting treatment.

Recommendations: For patients stably anticoagulated on warfarin (INR 2-4) and who are prescribed a single dose of antibiotics as prophylaxis against endocarditis, there is no necessity to alter their anticoagulants (grade C, level IV).

\section{Local haemostatic measures}

Tranexamic acid binds to plasminogen and inhibits subsequent lysis of fibrin. Orally administered tranexamic acid does not appear in saliva at detectable levels ${ }^{12}$ but as a mouthwash, the concentration of tranexamic acid remains sufficiently high to inhibit fibrinolysis for hours but insignificant levels are detected in the plasma.

In the UK, tranexamic acid mouthwashes are not readily available in the primary care setting and dentists cannot currently prescribe them. Four studies have evaluated the role of alternative treatments in patients undergoing dental extractions to secure haemostasis. However, in two of these studies tranexamic acid was also used to secure haemostasis.

Blinder et al. ${ }^{13}$ in a study of 150 patients undergoing dental extractions and without interruption of their anticoagulants, randomised patients to one of three groups: group 1 (50 patients mean INR 2.38) who were treated with gelatin sponges and sutures; group 2 (50 patients mean INR 2.7) who were treated with gelatin sponges, sutures and tranexamic acid mouthwashes and group 3 (50 patients mean INR 2.19) who received fibrin glue, gelatin sponges and sutures. Thirteen patients (8.6\%) developed post-operative bleeding: three in group 1, six in group 2 and four in group 3. The use of gelatin sponges and sutures provided adequate haemostasis for dental extraction without interruption of anticoagulants. Tranexamic acid mouthwashes or fibrin glue offered no benefit over resorbable gelatin sponges plus suturing.

In a subsequent study of 249 patients undergoing 543 dental extractions without interruption of their anticoagulant therapy $^{14}$ patients were divided into five groups according to their INR at the time of surgery. Local haemostasis in all groups was achieved with gelatin sponges and silk sutures. Overall, 12\% developed post-operative bleeding but the incidence of bleeding was not significantly different between group I (59 patients: mean INR 1.74) and group V (23 patients: mean INR 3.8).

In a study of 26 patients undergoing dental extraction with an INR of 2.0-4.2, the sockets were packed with a resorbable oxycellulose dressing ('Surgicel') and sutured with a resorbable suture. ${ }^{20}$ A second group of patients (INR 2.14.1) received local packing of the sockets with a fibrin adhesive ('Beriplast'). Only one patient in the second group had significant postoperative haemorrhage. The authors concluded that fibrin adhesive is as effective as oxycellulose dressing in preventing post-extraction haemorrhage in patients receiving oral anticoagulants.

In a Spanish study of 150 patients on oral anticoagulants undergoing dental extraction (229 sessions/367 dental extractions) and with an INR of 2-3 (145 cases 2-2.5; 84 cases 84 2.5-3) haemostasis was secured using haemostatic gauze and/or sutures or a local compression. ${ }^{15}$ In over $99 \%$ of cases the use of resorbable gelatin sponges plus sutures at the time of dental surgery was sufficient to achieve haemostasis and only one case of severe bleeding was observed. However, all patients received tranexamic acid mouthwashes every six hours for two days.

In 2003 Zanon and colleagues ${ }^{16}$ reported the results of a prospective study of 250 anticoagulated patients with INR values between 1.8-5.0 and 265 patients who were not receiving anticoagulation undergoing dental surgery. In all groups receiving oral anticoagulants, local haemostasis was secured with oxidised cellulose and silk sutures plus local application of a tranexamic acid saturated gauze swab for 30-60 minutes following surgery. There was 
no difference in bleeding complications between the two groups (4/250 for the anticoagulated group and $3 / 250$ in the control group). When the anticoagulated group was stratified according to INR (1.8-2, 2-3 and 3-4) bleeding complications were seen in $1.2 \%, 1.3 \%$ and $4.8 \%$ of patients respectively but this difference was not significantly different.

Five randomised trials have evaluated the role of tranexamic acid or other local haemostatic measures in patients undergoing oral surgery on continuous anticoagulant therapy.

In a study of 39 patients receiving oral anticoagulants for a cardiac valvular stenosis, a prosthetic heart valve or a vascular prosthesis, surgery was performed without a change in anticoagulation, but before suturing the operative field was irrigated in 19 patients with $10 \mathrm{ml}$ of a $4.8 \%$ solution of tranexamic acid and in 20 patients with placebo. ${ }^{17}$ Patients were instructed to rinse their mouths with $10 \mathrm{ml}$ of placebo or tranexamic acid for two minutes, four times a day for 7 days. The range of INRs was estimated at between 2.5-4.8. There was a statistically significant difference between the two groups with $8 / 20$ (40\%) patients in the placebo group having a total of 10 postoperative bleeds compared to only $1 / 19(5 \%)$ patients in the tranexamic acid-treated group.

In a second study, 93 patients undergoing invasive dental surgery were randomised to either tranexamic acid mouthwashes $(4.8 \%$ four times a day for seven days) (44 patients) or placebo (45 patients)..$^{18}$ Anticoagulants in this study were assessed by measuring plasma factor $\mathrm{X}$ levels and there were no differences in factor $X$ levels between the two groups of patients. In the group that received placebo mouthwashes, ten patients (22\%) had bleeding problems requiring treatment but none in the tranexamic acid group, a difference that was statistically significant.

A further study randomised 49 patients (undergoing 152 dental extractions) to either tranexamic acid (4.8\% four times a day for seven days) (26 patients: mean INR 3.0 range 2.3-4.0) or autologous fibrin glue (23 patients: mean INR 3.1 range 2.1-4.0)..$^{19}$ Two cases of post-operative bleeding were noted in the group that received autologous fibrin glue but both cases had elevated INRs (5.9/7.9) at the time of surgery. Tranexamic acid was found to be a more cost-effective alternative.

In a study of 85 patients on warfarin undergoing dental surgery randomised to tranexamic acid mouthwashes (two minutes four times a day: mean INR 2.7) for either two days or five days (mean INR 2.8). ${ }^{19}$ There were no significant differences in bleeding complications between the two groups.

Sacco et al. reported a significant difference in bleeding complications between a group of patients whose warfarin dose was reduced 72 hours prior to surgery compared to those whose warfarin continued uninterrupted but who received tranexamic acid mouthwashes for six days. The rate of bleeding was $15 \%$ in the first group compared to $9 \%$ in the second group.

The current ACCP guidelines recommend the use of tranexamic acid mouthwashes (or epsilon amino caproic acid (EACA) mouthwashes) without interruption of anticoagulant therapy in anticoagulated patients undergoing dental surgery. ${ }^{20}$

Recommendations: In patients undergoing dental extractions, bleeding may be minimised by:

a. The use of oxidised cellulose ('Surgicel') or collagen sponges and sutures (grade B, level IIb)

b. 5\% tranexamic acid mouthwashes used four times a day for 2 days (grade A, level Ib). Tranexamic acid is not readily available in the primary care dental setting.

\section{When should the INR be measured before} a dental procedure?

In patients receiving long-term anticoagulant therapy and who are stably anticoagulated on warfarin an INR check 72 hours prior to surgery is recommended. This allows sufficient time for dose modification if necessary to ensure a safe INR (2-4) on the day of dental surgery.

There is no need to check the INR for non-invasive dental procedures although scaling subgingivally will require an INR check.

The INR should also be checked if performing an inferior alveolar nerve block (IANB) as there is an anecdotal risk of haematoma and airway compromise. If needed, an IANB should be given cautiously, using an aspirating syringe, with an INR <3.0.
8. The use of anti-inflammatory drugs in patients receiving warfarin and undergoing dental surgery

Non-steroidal anti-inflammatory drugs (NSAIDs) are avoided in patients receiving oral anticoagulants because of their anti-platelet action and the risk of over anticoagulation and haemorrhage. Cyclo oxygenase-2 (COX-2) selective inhibitors are perceived to be safer than conventional NSAIDs in patients receiving warfarin and this has led to an increase in their use in such cases. However, there is accumulating data that COX-2 inhibitors can cause an increase in INR and recent studies highlighting that the risk of upper GI haemorrhage are similar in warfarin users concomitantly taking either non-selective NSAIDs or COX-2 inhibitors.

Recommendations: Patients taking warfarin should not be prescribed nonselective NSAIDs and COX-2 inhibitors as analgesics following dental surgery (grade B, level IIb.)

\section{Disclaimer}

While the advice and information in these guidelines is believed to be true and accurate at the time of going to press, neither the authors, the British Society for Haematology nor the publishers accept any legal responsibility for the content of these guidelines.

Date for guideline review: September 2011

For their helpful comments and suggestions, we are grateful to $\mathrm{Mr}$ Andrew Brewer in the Department of Oral and Maxillofacial Surgery, Glasgow Royal Infirmary and Pippa K Blacklock in the Department of Oral and Maxillofacial Surgery, Derriford Hospital, Plymouth.

1. Ziffer A M, Scopp I W, Beck J, Baum J, Berger A R. Profound bleeding after dental extractions during dicumarol therapy. N Engl J Med 1957; 256: 351-353.

2. Wright IS, Beck D F, Marple C D. Myocardial infarction and its treatment with anticoagulants; summary of findings in 1031 cases. Lancet 1954; 266: 92-95.

3. Kirkwood T B. Calibration of reference thromboplastins and standardisation of the prothrombin time ratio. Thromb Haemost 1983; 49: 238-244.

4. Randall C. Surgical management of the primary care dental patient on warfarin. Dent Update 2005; 32: 414-416, 419-420, 423-424 (passim).

5. Al-Mubarak S, Rass M A, Alsuwyed A, Alabdulaaly A, Ciancio S. Thromboembolic risk and bleeding in patients maintaining or stopping oral anticoagulant therapy during dental extraction. J Thromb Haemost 2006; 4: 689-691.

6. Wahl M J. Dental surgery in anticoagulated patients. Arch Intern Med 1998; 158: 1610-1616.

. Devani P, Lavery K M, Howell C J. Dental extractions in patients on warfarin: is alteration of anticoagulant regime necessary? Br J Oral Maxillofac Surg 1998; 36: 107-111.

8. Campbell J H, Alvarado F, Murray R A. Anticoagulation and minor oral surgery: should the anticoagulation regimen be altered? J Oral Maxillofac Surg 2000; 58: 131-136.

9. Evans I L, Sayers M S, Gibbons A J, Price G, Snooks $H$, Sugar A W. Can warfarin be continued during 
dental extraction? Results of a randomized controlled trial. Br J Oral Maxillofac Surg 2002; 40: 248-252.

10. Sacco R, Sacco M, Carpenedo M, Moia M. Oral surgery in patients on oral anticoagulant therapy: a randomized comparison of different INR targets. J Thromb Haemost 2006; 4: 688-689.

11. Gould FK, Elliott TS, Foweraker J et al. Guidelines for the prevention of endocarditis: report of the Working Party of the British Society for Antimicrobial Chemotherapy. J Antimicrob Chemother 2006: 57: 1035-1042.

12. Sindet-Pedersen $\mathrm{S}$. Distribution of tranexamic acid to plasma and saliva after oral administration and mouth rinsing: a pharmacokinetic study. J Clin Pharmacol 1987; 27: 1005-1008.

13. Blinder D, Manor Y, Martinowitz U, Taicher S, Hashomer T. Dental extractions in patients maintained on continued oral anticoagulant: comparison of local hemostatic modalities. Oral Surg Oral Med Oral Pathol Oral Radiol Endod 1999; 88: $137-140$.

14. Blinder D, Manor Y, Martinowitz U, Taicher S. Dental extractions in patients maintained on oral anticoagulant therapy: comparison of INR value with occurrence of postoperative bleeding. Int J Oral Maxillofac Surg 2001; 30: 518-521.

15. Vicente Barrero M, Knezevic M, Tapia Martin M et al. Oral surgery in patients undergoing oral anticoagulant therapy. Med Oral 2002; 7: 63-66, 67-70.

16. Zanon E, Martinelli F, Bacci C, Cordioli G, Girolami A. Safety of dental extraction among consecutive patients on oral anticoagulant treatment managed using a specific dental management protocol. Blood Coagul Fibrinolysis 2003; 14: 27-30.

17. Sindet-Pedersen $S$, Ramstrom G, Bernvil S, Blomback M. Hemostatic effect of tranexamic acid mouthwash in anticoagulant-treated patients undergoing oral surgery. N Engl J Med 1989; 320: 840-843.

18. Ramstrom G, Sindet-Pedersen S, Hall G, Blomback $\mathrm{M}$, Alander U. Prevention of postsurgical bleeding in oral surgery using tranexamic acid without dose modification of oral anticoagulants. J Oral Maxillofac Surg 1993; 51: 1211-1216.

19. Carter G, Goss A. Tranexamic acid mouthwash - a prospective randomized study of a 2-day regimen vs 5 -day regimen to prevent postoperative bleeding in anticoagulated patients requiring dental extractions. Int J Oral Maxillofac Surg 2003; 32: 504-507.

20. Ansell J, Hirsh J, Poller L, Bussey H, Jacobson A, Hylek E. The pharmacology and management of the vitamin K antagonists: the Seventh ACCP Conference on Antithrombotic and Thrombolytic Therapy. Chest 2004; 126; 3 (Suppl): 204S-233S.

\section{Erratum}

\section{Intravenous conscious sedation in children for outpatient dentistry (BDJ 2007; 203: 323-331)}

It has been brought to our attention that Table 4 in the above paper by Mikhael, Wray and Robb was printed incorrectly. The correct version of the table is given below. We wish to apologise to the authors and to readers for this error and any confusion and inconvenience that may have been caused.

\section{Table 4 Verbal contact after sedation}

\begin{tabular}{|l|l|}
\hline $\begin{array}{l}\text { Verbal contact maintained } \\
\text { throughout }\end{array}$ & $994(99.4 \%)$ \\
\hline $\begin{array}{l}\text { Verbal contact not maintained } \\
\text { throughout }\end{array}$ & $6(0.6 \%)$ \\
\hline
\end{tabular}

DOI: $10.1038 /$ bdj.2007.915 